10

\title{
Super-achromatic Quarter-wave Phase Retarder for Visible, Near Infrared and Short Wave Infrared Region Applications*
}

\author{
(C) N. Mukhopadhya ${ }^{1}$, A. Saha ${ }^{2}$, and K. Bhattacharya ${ }^{3}$ \\ ${ }^{1}$ Department of Electronics \& Communication Engineering, Global Institute of Management and Technology, Krishnagar, \\ 741102 Nadia, India \\ ${ }^{2}$ Department of Electronics \& Communication Engineering, B. P. Poddar Institute of Management and Technology, \\ 700052 Kolkata, India \\ ${ }^{3}$ Department of Applied Optics \& Photonics, University of Calcutta, \\ JD-2, Sector 3, Salt Lake City, Kolkata 700098, India \\ e-mail: nilu.opt@gmail.com
}

Received December 08, 2019

Revised May 07, 2020

Accepted May 07, 2020

Phase retarders are essential components in optical system design as they can control the phase shifts associated with the vector components of incoming light waves and hence, decide the state of polarization. Different optical systems are designed to work at different wavelength spectrums. Most of these developments focus on the different wavelength regions between visible to infrared, of which the near infrared and short-wave infrared ranges are popular domains for defense and remote sensing applications. In this present communication, a super achromatic quarterwave phase retarder consisting of four birefringent plates of different materials, i.e. KDP, crystalline quartz, ADP and $\mathrm{ZnS}$ has been proposed, to cover the visible, near infrared and short wave infrared regions from 400-2100 nm. As circularly polarized light is less affected by atmospheric conditions the use of an achromatic broadband quarterwave phase retarder as proposed is quite useful. The proposed system shows acceptable performance over the intended range of wavelengths.

Keywords: Wave plates, Super achromatic, Retarder, SWIR.

* Полный текст статьи опубликован в „Optics and Spectroscopy“ 2020 V. 128. N 8. 